\title{
Modélisation de l'eutrophisation de la Baie de Vilaine et des phénomènes d'anoxie associés
}

\author{
Annie Chapelle \\ IFREMER - Centre de Brest \\ B.P. 70 - 29263 Plouzané
}

1.
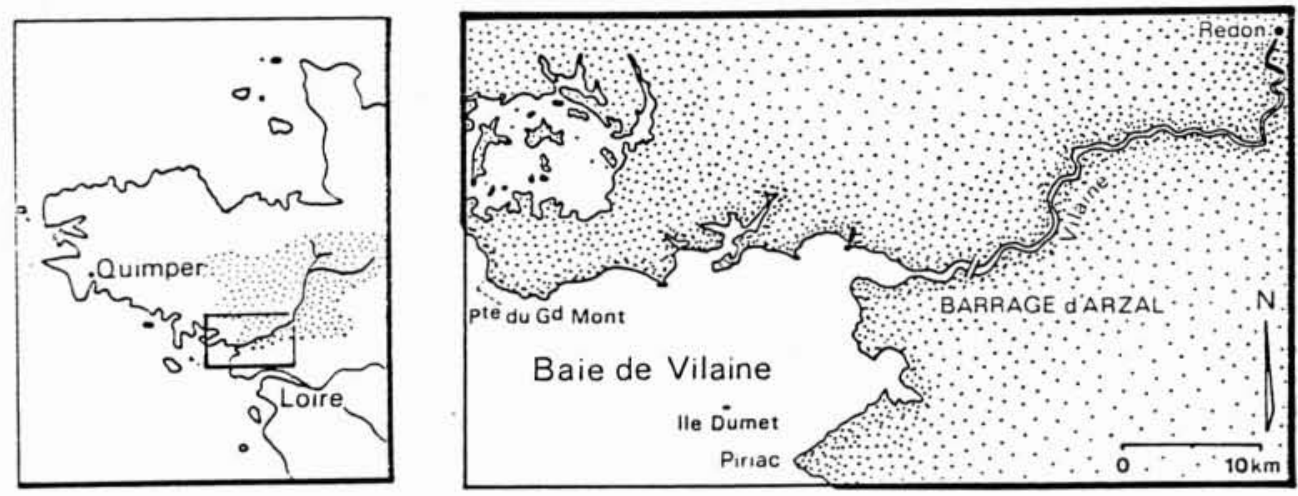

La baie de Vilaine, située au nord de la Loire, a une superficie de $200 \mathrm{~km}^{2}$ et son bassin versant drainé par le fleuve Vilaine et son affluent l'Oust s'étend sur un tiers de la Bretagne (fig. 1). La grande activité agricole de cette région, et les rejets urbains et industriels des villes traversées (Rennes, Redon) sont responsables de teneurs élevées en azote et en phosphore dans le fleuve, le classant à la limite supérieure de l'eutrophie (QUEGUINER, 1988). La construction du barrage d'Arzal (1970), prévu pour assécher la zone marécageuse autour de Redon et établir une réserve d'eau potable à réduit l'estuaire d'une quarantaine de kilomètres. Il en résulte un déplacement des fonctions de dégradation de la matière organique vers la baie (MERCERON, 1986) ainsi qu'une stratification plus grande de l'estuaire. Enfin dans la baie, la faiblesse des courants de marées favorise une stratification thermohaline et par temps calme les temps de résidence peuvent être élevés (SAlomon et LAZURE, 1988).

Ces caractéristiques écologiques particulières expliquent la fragilité de l'écosystème de la baie, en cours d'eutrophisation comme le témoignent différentes manifestations chroniques: eaux colorées, déficit estival en oxygène près du fond, proliférations de Dinophysis, marées vertes.

En juillet 1982, plusieurs dizaines de tonnes de poissons morts par asphyxie ont été récoltés dans la baie. Une commission fut alors constituée chargée de l'étude de ces problèmes et de la recherche de solutions concrètes pour restaurer la qualité des eaux d'une région oủ la pêche, la conchyliculture et le tourisme sont des enjeux économiques importants.

La mise en place d'un modèle mathématique de l'écosystème de la baie est à considérer comme un outil de synthèse des données acquises, de compréhension des mécanismes élémentaires; il permet de tester différentes modalités de la lutte contre l'eutrophisation.

\section{Eutrophication modelling in the Vilaine Bay. An application to the anoxia events}

The Vilaine bay (South Brittany) has been showing for many years eutrophication troubles (red tides, anoxia, toxic blooms...). Facing these biological and economical problems, scientific studies have been performed and the results are joined in an ecological box model of the bay. It simulates the primary production through nitrogen cycle, dissolved oxygen, and salinity, used to calibrate the advection-dispersion fluxes of the physical submodel. The model is running several years to bring out the seasonal characteristics of this site. A special attention is reported on the oxygen balance sheet. Simulations try to quantify the oxygen demand and to recognize the major cause of anoxia. These results are applied to reproduce the July 1982 anoxia, which caused drastic fish mortalities. 


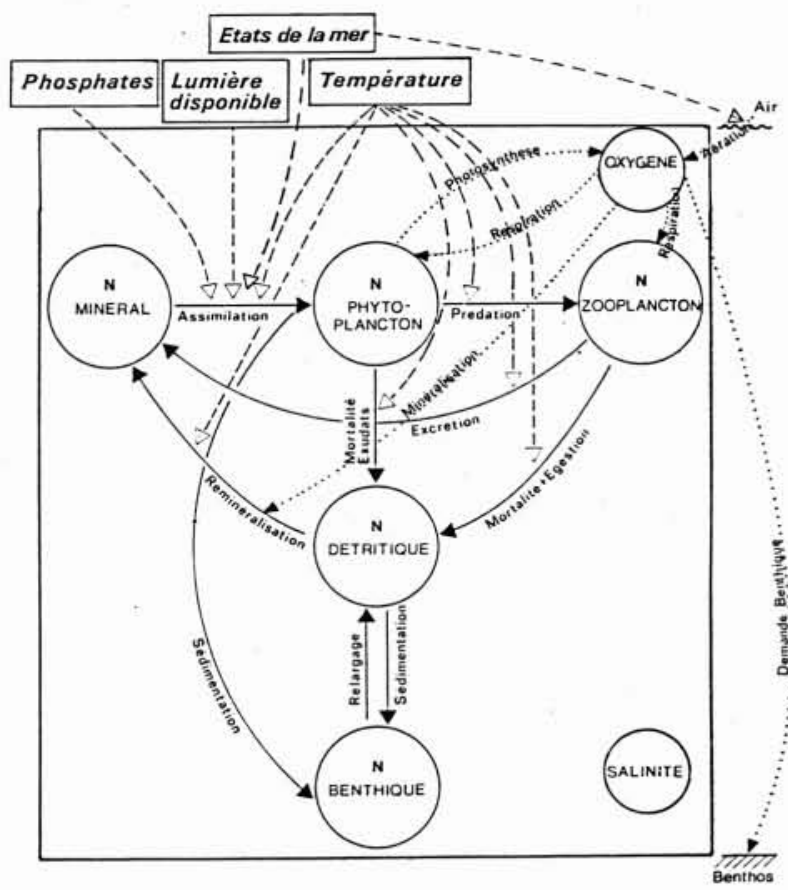

2. Schéma conceptuel du modèle biologique.

O variables d"état

$\square$ variables forçantes

$\rightarrow$ flux d'azote

.... flux d'oxygène

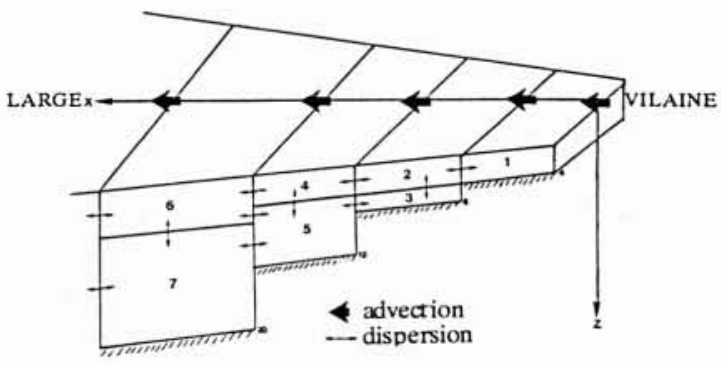

3. Modèle en boîtes.

$\rightarrow$ Advection

$\leftarrow$ Dispersion

\section{Conception du modèle}

Afin de représenter l'évolution saisonnière des sels nutritifs et de la production primaire, le cycle de l'azote est modélisé et appliqué à la baie au moyen d'un découpage spatial en boîtes.

\section{Modèle biologique}

Il est constitué de sept variables d'état (fig. 2):

- 5 variables azotées correspondant aux principales formes de l'azote en milieu côtier ;

- l'oxygène dissous, élément-clé pour la qualité des eaux ; - la salinité, traceur conservatif utilisé pour calibrer les flux dans le modèle physique.

Le système est par ailleurs influencé par quatre variables forçantes :

- la température, accélérateur de nombreuses réactions enzymatiques ;

- la lumière, indispensable pour la photosynthèse ;

- le phosphore, élément-limitant, avec l'azote, la croissance du phytoplancton (la simulation du cycle du phosphore en parallèle à celui de l'azote sera entreprise seulement dans une phase ultérieure, en raison des échanges complexes eau-sédiment ;

- l'état de la mer, paramètre météorologique représentant l'effet du vent. Il modifie les échanges d'oxygènes à la surface et induit une remise en suspension, qui augmente le coefficient d'extinction lumineuse.

L'évolution temporelle de chaque variable est décrite sous forme d'équation différentielle, somme des processus de pertes et de gains (détails en annexe).
$d(\mathrm{~N}$ minéral $) / d t=$ minéralisation + excrétion zooplanctonique - assimilation phytoplanctonique

$d($ phytoplancton $) / d t=$ croissance - mortalité - sédimentation - broutage

$d($ zooplancton $) / d t=$ croissance - mortalité - excrétion $d(\mathrm{~N}$ détritique $) / d t=$ mortalité $($ phyto + zoo $)+$ relargage - minéralisation - sédimentation $d(\mathrm{~N}$ benthique $) / d t=$ sédimentation (phyto $+\mathrm{N}$ détritique) - relargage

$d\left(\mathrm{O}_{2}\right) / d t=$ aération + photosynthèse - respiration (phyto + zoo) - minéralisation - demande benthique.

La formulation mathématique de ces mécanismes repose sur des recherches bibliographiques, des mesures in situ et parfois sur des relations arbitraires simples telles que la dépendance linéaire entre le coefficient d'extinction lumineuse non chlorophyllien et l'état de la mer.

\section{Modèle physique}

Le découpage spatial en boîtes est réalisé selon les gradients (salinité, sels nutritifs...) côte-large, et surfacefond (fig. 3).

Les flux advectifs correspondent aux apports d'eau douce du fleuve dans les boîtes de surface. Les courants résiduels de marée de faible amplitude sont négligés (SALOMON et LAZURE, 1988). L'effet instantané de la marée, l'influence du vent et la stratification sont pris en compte dans les termes de dispersion verticale. La dispersion horizontale est constante et calibrée d'après les données de salinité. Les conditions aux limites du large posent un problème. Elles sont mal connues et peuvent être influencées par la Loire lorsque celle-ci est en crue. Pour le moment des valeurs saisonnières du plateau continental breton sont employées (LeCORRE et Treguer, 1976). 

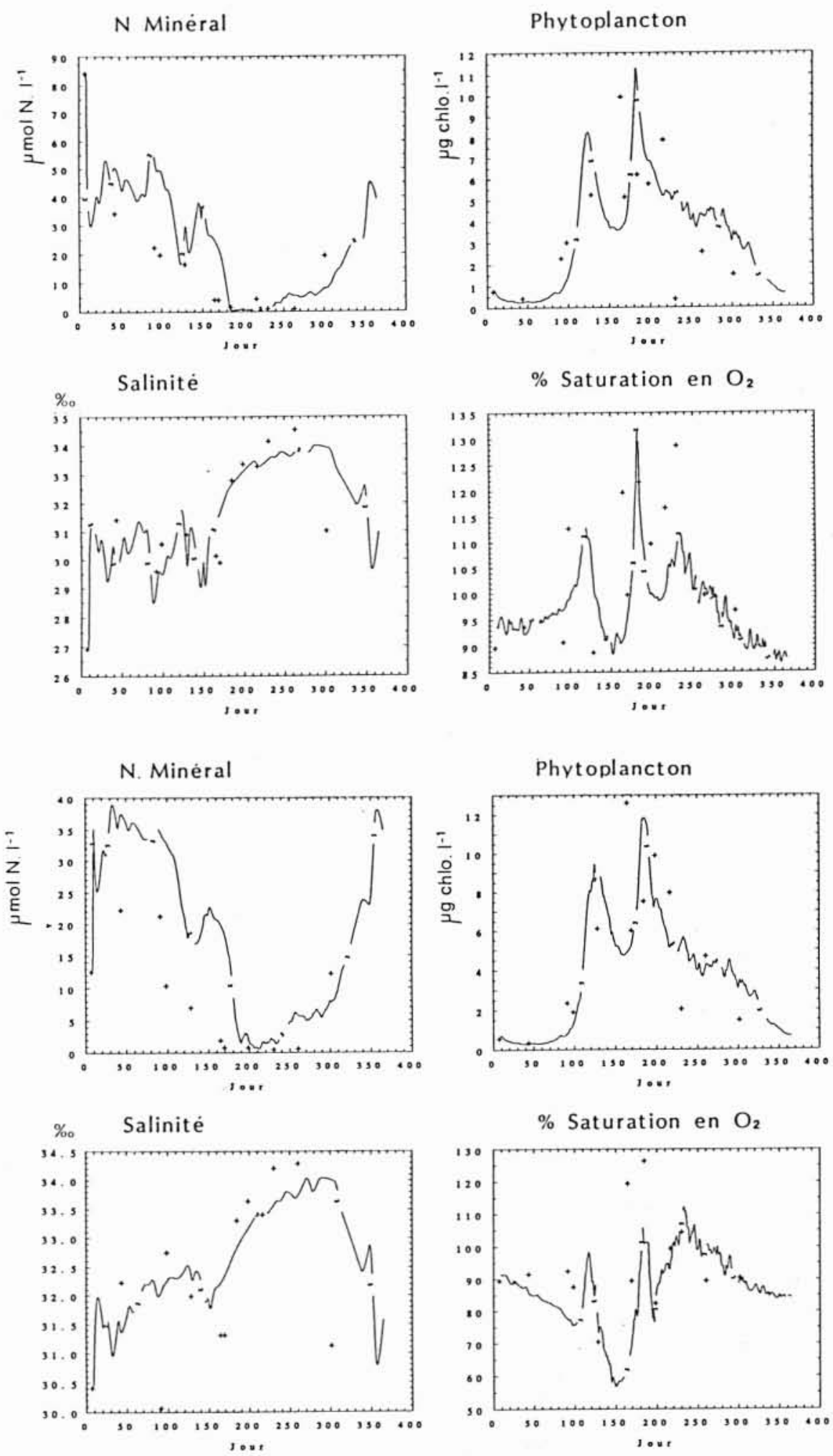

4. Simulation pour l'année 1984 et les boites 2 (surface) et 3 (fond).

+ données

- simulation. 


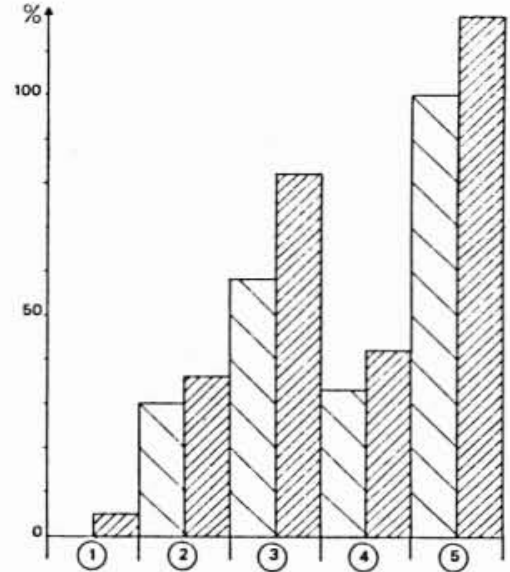

5. \% de variation des minima en oxygène dans les boittes de fond par rapport à la simulation 1984.

Boite 3

Boîte 5

1 sans respiration zooplanctonique

2 sans demande benthique

3 sans respiration phytoplanctonique

4 sans reminéralisation

5 sans consommation de l'eau (respiration-reminéralisation).

\section{Réalisation du modèle}

Mise en auvre informatique: Les équations différentielles sont intégrées par une méthode numérique de type Runge Kutta à pas variable. Les flux physiques sont discrétisés. L'ensemble du logiciel, écrit en Fortran, est une adaptation du logiciel ELISE (Menesguen, à paraître).

Calibration: La mise en équation du modèle fait intervenir un lot de paramètres dont les valeurs sont imprécises (dans une gamme donnée). Ces dernières sont estimées au moyen d'une comparaison visuelle simulation/données (fig. 4) pour l'année 1984, année où les mesures ont été les plus nombreuses. Pour les variables zooplanctoniques, détritiques et benthiques, l'absence de connaissance in situ entraîne une comparaison avec d'autres sites. Par ailleurs de nouvelles campagnes d'échantillonnage sont prévues.

Vérification du modèle: Une fois calibré, le modèle est appliqué aux années $83,84,85$ où un suivi écologique a été mené en baie de Vilaine.

L'évolution saisonnière présente une tendance générale : la production primaire est directement influencée par les apports nutritifs du fleuve, avec un premier pic printanier lorsque les conditions météorologiques deviennent favorables, puis des blooms estivaux suivant les crues. Pendant la croissance du phytoplancton, des sursaturations en surface apparaissent, alors qu'au moment du déclin, la quantité d'oxygène au fond chute. Cette opposition est accentuée par temps calme du fait de la stratification. Au contraire, l'agitation de la mer tend à homogénéiser le volume d'eau et à réduire les hypoxies.

\section{Application du modèle aux phénomènes d'hypoxie, anoxie}

Analyse de sensibilité du bilan d'oxygène: Différents processus étant responsables de la consommation d'oxygène, l'importance quantitative de chacun peut être testée pour le modèle. A chaque simulation, un des mécanismes est ignoré, et les minima d'oxygène d'été sont comparés à la simulation témoin (1984) (fig. 5).

Il ressort de cette étude que la consommation d'eau est nettement supérieure à celle des sédiments, ce qui corrobore l'hypothèse de MERCERoN (1987), la respiration du phytoplancton étant le phénomène majeur. Pour réduire la probabilité d'apparition d'anoxie aussi bien que pour limiter les hypoxies estivales (nocives pour les poissons), une réduction de la biomasse phytoplanctonique serait souhaitable.

Un autre facteur, sur lequel aucune action n'est envisageable, est impliqué dans les chutes d'oxygène: la stratification verticale. Selon la situation météorologique, le mélange vertical peut être fortement réduit, interdisant le réapprovisionnement en oxygène des couches du fond. Une telle situation est apparue en juillet 1982.

Simulation de l'anoxie de juillet 82: Des dizaines de tonnes de poissons morts ou moribonds se sont échoués à la fin du mois de juillet. Quelques jours auparavant le débit 


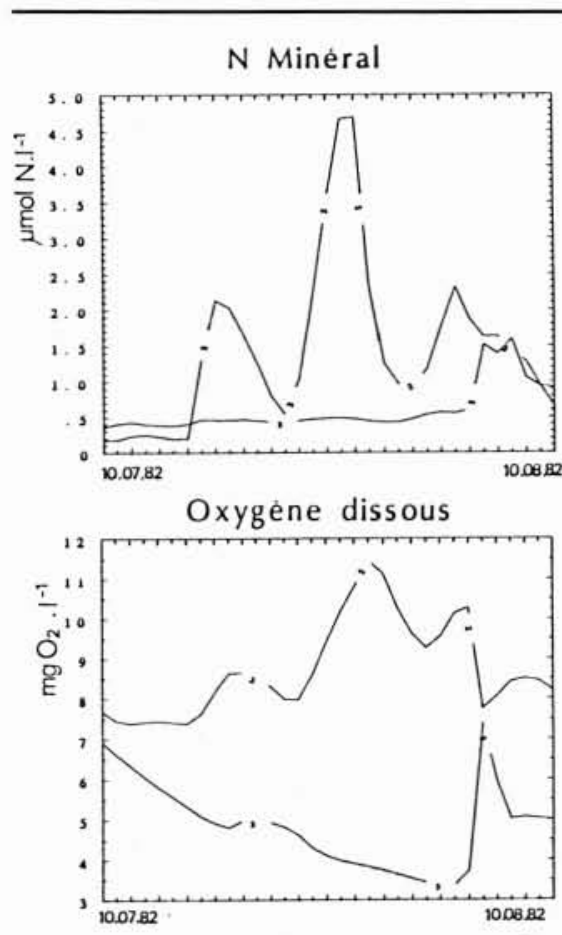

\section{Conclusion}

Cette première étape dans la réalisation d'un modèle d'eutrophisation de la baie de Vilaine permet déjà de représenter les caractéristiques fondamentales de cet écosystème fortement influencé par les apports du fleuve, et ses réactions en cas de rupture de l'équilibre écologique. Par la suite, une étude quantitative de la relation biomasse phytoplanctonique, apports terrestres de sels nutritifs sera menée en simulant le cycle du phosphore en parallèle à celui de l'azote. Le modèle testera alors différents scenarii d'épuration des eaux fluviales et leurs conséquences dans le domaine côtier. Celles-ci pourront être comparées aux effets de déphosphatation simulés sur le cours terrestre du fleuve (BETURE-SETAME, 1988).

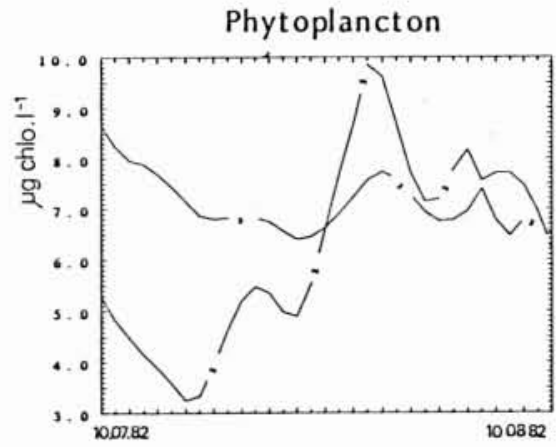

7. Simulation de l'oxygène du 10 juillet (jour 190) au 10 août (jour 222) 1982.

de la Vilaine avait fortement augmenté, le temps restant calme par ailleurs (fig. 6). La conjonction de ces deux phénomènes a vraisemblablement entraîné l'établissement d'une forte halocline limitant les échanges surface-fond. Prenant en compte ces facteurs météorologiques le modèle simule l'évolution de l'oxygène dissous du 10 juillet au 10 août (fig. 7).

L'arrivée d'eau douce enrichit le milieu et stimule le développement du phytoplancton. Au fond les échanges sont réduits, la matière organique sédimente et consomme de l'oxygène par reminéralisation, le phytoplancton ne reçoit plus assez de lumière pour photosynthétiser et la respiration devient dominante. La baisse des teneurs en oxygène correspond à l'époque où les mortalités de poissons sont apparues mais elle fut certainement de plus grande amplitude. L'équilibre n'est rétabli qu'à la faveur des coups de vent du début août.

\section{Références bibliographiques}

Beture-Setame (1988). - Plan de lutte contre la limitation des flux de nutriments en aval de la Vilaine. Rapport final, $110 \mathrm{p}$.

Lecorre P. et Treguer P. (1976). - Contribution à létude de la matière organique dissoute et des sels nutritifs dans l'eau de mer. Caractéristiques chimiques du golfe de Gascogne et des upwelling côtiers de l'Afrique du nord-ouest. Thèse d'état es-Sciences, UBO, 490 p.

Merceron M. (1986). - Etude de la matière organique et demande en oxygène des sédiments en baie de Vilaine. Rapport IFREMER DERO-86.20-EL, $40 \mathrm{p}$.

MERCERON M. (1987). - Mortalités de poissons en baie de Vilaine (juillet 1982). Causes. Mécanismes. Propositions d'action. Rapport IFREMER DERO-87.14-EL, $100 \mathrm{p}$.

QUeGuiner B. (1988). - Synthèse scientifique du programme pluriannuel 1983-1988 de la commission quadripartite chargée de l'aménagement halieutique et de la protection hydrobiologique de la baie de Quiberon-Vilaine, $134 \mathrm{p}$.

SAlomon J.C. et Lazure P. (1988). - Etude par modèle mathématique de quelques aspects de la circulation marine entre Quiberon et Noirmoutier. Rapport IFREMER DERO88.26-EL, $104 \mathrm{p}$. 
Système d'équations différentielles et tableau de paramètres

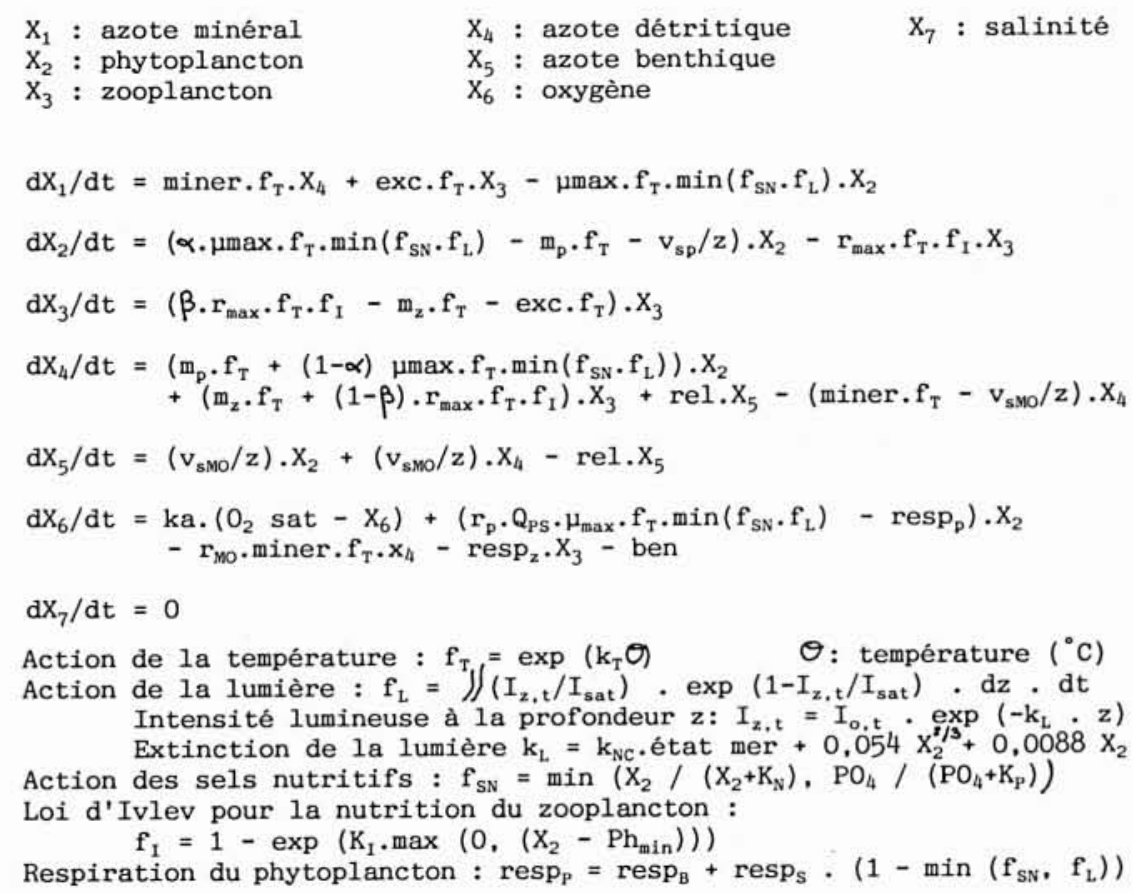

\begin{tabular}{|c|c|c|c|}
\hline Paramètres & Définition & Unité & Valeur \\
\hline$\mu \max$ & $\begin{array}{l}\text { Taux maximal de croissance du phytoplancton } \\
\text { à } 0^{\circ} \mathrm{C}\end{array}$ & $j^{-1}$ & 0,45 \\
\hline $\mathrm{k}_{\mathrm{T}}$ & $\begin{array}{l}\text { Taux d'augmentation des vitesses avec la } \\
\text { température }\end{array}$ & ${ }^{\circ} \mathrm{C}^{-1}$ & 0.07 \\
\hline Isat & Intensité lumineuse optimale & $\mathrm{Wm}^{-2}$ & 70 \\
\hline $\mathrm{K}_{\mathrm{N}}$ & $\begin{array}{l}\text { Constante de Michaelis pour 1'absorption } \\
\text { d'azote }\end{array}$ & $\mu \mathrm{mol} \mathrm{N} \cdot \mathrm{I}^{-1}$ & 4 \\
\hline $\mathrm{K}_{\mathrm{P}}$ & $\begin{array}{l}\text { Constante de Michaelis pour 1'absorption } \\
\text { du phosphore }\end{array}$ & $\mu \mathrm{mol} P .1^{-1}$ & 0.5 \\
\hline$\alpha$ & Assimilation du phytoplancton & sd & 0,8 \\
\hline$m^{b}$ & Taux de mortalité du phytoplancton à $0^{\circ} \mathrm{C}$ & $j^{-1}$ & 0,005 \\
\hline $\mathrm{v}_{\mathrm{sp}}$ & Vitesse de sédimentation du phytoplancton & $\mathrm{m} \cdot \mathrm{j}^{-1}$ & 0,5 \\
\hline $\mathrm{Ph}_{\min }$ & $\begin{array}{l}\text { Seuil d'échappement du phytoplancton à la } \\
\text { prédation }\end{array}$ & $\mu \mathrm{mol} \mathrm{N} .1^{-1}$ & 0,1 \\
\hline $\mathrm{r}_{\mathrm{p}}$ & $\begin{array}{l}\text { Rapport oxygène/azote pour la photosynthèse } \\
\text { et la respiration du phytoplancton }\end{array}$ & $\mathrm{mgO}_{2} \cdot \mu \mathrm{molN} \mathrm{N}^{-1}$ & 0,212 \\
\hline $\mathrm{Q}_{\mathrm{PS}}$ & Quotient photosynthèse & sd & 1,2 \\
\hline $\operatorname{resp}_{\mathrm{B}}$ & Respiration basale du phytoplancton & $j^{-1}$ & 0,03 \\
\hline resps $_{\mathrm{s}}$ & Respiration du phytoplancton liée au stress & $j^{-1}$ & 0.05 \\
\hline$r_{\max }$ & Taux maximal d'ingestion du zooplancton à $0^{\circ} \mathrm{C}$ & $j^{-1}$ & 0,25 \\
\hline $\mathrm{K}_{\mathrm{I}}$ & $\begin{array}{l}\text { Constante d'Ivlev pour l'ingestion du zoo- } \\
\text { plancton }\end{array}$ & 1. $\mu \mathrm{mol} \mathrm{N} \mathrm{N}^{-1}$ & 0,02 \\
\hline$\beta$ & Assimilation du zooplancton & sd & 0,6 \\
\hline $\operatorname{exc}$ & Taux d'excrétion du zooplancton à $0^{\circ} \mathrm{C}$ & $j^{-1}$ & 0,01 \\
\hline $\begin{array}{l}\mathrm{m}_{\mathrm{z}} \\
\text { resp }_{\mathrm{z}}\end{array}$ & $\begin{array}{l}\text { Taux de mortalité du zooplancton à } 0^{\circ} \mathrm{C} \\
\text { Respiration du zooplancton }\end{array}$ & $\mathrm{j}^{-1} \cdot \mu \mathrm{mol} \mathrm{N}^{-1} \cdot \mathrm{j}^{-1}$ & $\begin{array}{l}0,005 \\
0,0084\end{array}$ \\
\hline miner & $\begin{array}{l}\text { Taux de minéralisation de } 1^{\prime} \text { azote détri- } \\
\text { tique à } 0^{\circ} \mathrm{C}\end{array}$ & $j^{-1}$ & 0,02 \\
\hline $\begin{array}{l}v_{\text {smo }} \\
\text { rel }\end{array}$ & Vitesse de sédimentation de l'azote détritique & $\mathrm{m} \cdot \mathrm{j}^{-1}$ & 1 \\
\hline $\begin{array}{l}\mathrm{rel} \\
\mathrm{r}_{\mathrm{m}}\end{array}$ & $\begin{array}{l}\text { Taux de relargage de } l^{\prime} \text { azote benthique } \\
\text { Rapport oxygène/azote pour la minéralisation }\end{array}$ & $\mathrm{mgO}_{2}^{-1} \cdot \mu \mathrm{mol} \mathrm{N}^{-1}$ & $\begin{array}{l}0.07 \\
0.288\end{array}$ \\
\hline $\begin{array}{l}r_{m o} \\
\text { Ben }\end{array}$ & $\begin{array}{l}\text { Rapport oxygène/azote pour la minéralisation } \\
\text { Demande benthique en oxygène }\end{array}$ & $\mathrm{gO}_{2} \mathrm{~m}^{-2} \mathrm{j}^{-1}$ & 1 \\
\hline $\mathrm{K}_{\mathrm{sc}}$ & $\begin{array}{l}\text { Coefficient d'extinction lumineuse non } \\
\text { chlorophyllien }\end{array}$ & $\mathrm{m}^{-1}$ & 0,2 \\
\hline
\end{tabular}

